\title{
I'M THINKING OF A NUMBER ...
}

\author{
ADAM HAMMETT AND GREG OMAN
}

\begin{abstract}
Consider the following game: Player A chooses an integer $\alpha$ between 1 and $n$ for some integer $n \geq 1$, but does not reveal $\alpha$ to Player B. Player B then asks Player A a yes/no question about which number Player A chose, after which Player A responds truthfully with either "yes" or "no." After a predetermined number $m$ of questions have been asked $(m \geq 1)$, Player B must attempt to guess the number chosen by Player A. Player B wins if she guesses $\alpha$. The purpose of this note is to find, for every $m \geq 1$, all canonical $m$-question algorithms which maximize the probability of Player B winning the game (the notion of "canonical algorithm" will be made precise in Section 3$)$.
\end{abstract}

\section{Introduction}

I'm thinking of a number between 1 and 1000. I will allow you one guess, but prior to guessing you get to ask me a preliminary "yes/no" question about which number I chose, and I will answer truthfully. Clearly, you don't want to ask, "Is the number you picked between 1 and 1000?" as you already know that the answer is "yes." Similarly, you wouldn't ask, "Did you pick the numbers 3 and 298?" since (unless I choose not to abide by the rules of the game) you know the answer to this query is "no." So at the very least, you should ask me a question to which you do not already know the answer. Two such questions are listed below. Which of the following do you believe will yield a higher probability of guessing the number I chose (assuming you guess rationally after I give you my answer)?

$Q_{1}$ : "Did you choose number 1 ?", or

$Q_{2}$ : "Is the number you chose between 1 and 500?"

You're thinking $Q_{2}$ is the more prudent choice, right? After all, the answer to $Q_{1}$ is almost certainly "no," and (if it is indeed "no") then you'll have to choose from 999 numbers. But if you ask $Q_{2}$, you can immediately eliminate 500 numbers. Would you be surprised to discover that it doesn't matter which question you choose? Whether you ask me $Q_{1}$ or $Q_{2}$, the probability of correctly guessing my number is the same! 


\section{A. HAMMETT AND G. OMAN}

Let us briefly explain why this is the case. If you ask me $Q_{1}$, and I answer "yes," then you will guess number 1 and win. On the other hand, if you ask $Q_{1}$ and I answer "no," then you will just guess some integer between 2 and 1000. This means the probability of winning if you elect to ask $Q_{1}$ equals

$$
\frac{1}{1000} \cdot 1+\frac{999}{1000} \cdot \frac{1}{999}=\frac{2}{1000} .
$$

Notice that if you had guessed randomly with no preliminary question, then the probability of winning is clearly $\frac{1}{1000}$; thus asking $Q_{1}$ results in doubling the probability of finding my number. Now, similar reasoning reveals that electing to ask $Q_{2}$ instead delivers a probability of winning equal to

$$
\frac{500}{1000} \cdot \frac{1}{500}+\frac{500}{1000} \cdot \frac{1}{500}=\frac{2}{1000},
$$

and that's the same as before!

Now, let's change the rules a bit. Let's keep the same basic setup, but this time I will allow you a sequence of two preliminary questions. Specifically, I will permit you an initial yes/no question about my chosen number, and then a second yes/no question after I answer your first query. Following my answer to your second question, you will attempt to guess the number I picked. Which of the following two algorithms yields a higher probability that you will guess my number?

( 1) First question: "Did you choose number 1 or number 2?"

$\hookrightarrow$ If I say "yes," ask second question "Did you choose number 2?"

$\hookrightarrow$ If I say "no," ask second question "Did you choose number 3?", or

( 2) First question: "Did you choose a number between 1 and 500?"

$\hookrightarrow$ If I say "yes," ask second question "Did you choose a number between 1 and 250?"

$\hookrightarrow$ If I say "no," ask second question "Did you choose a number between 501 and 750?"

Perhaps unexpectedly, both algorithms yield the same probability (namely, $\frac{4}{1000}$ ) of guessing my number. However, the following related algorithm yields only a $\frac{3}{1000}$ probability of guessing correctly:

( 3 ) First question: "Did you choose number 1?"

$\hookrightarrow$ If I say "yes," then you know my number and don't need a second question.

$\hookrightarrow$ If I say "no," ask second question "Did you choose number 2 or number 3 ?" 


\section{I'M THINKING OF A NUMBER ...}

The purpose of this note is to generalize these surprising facts. In particular, after some formal preliminaries in the next section, we shall describe all "natural" $m$-question algorithms which deliver the maximum probability of Player B guessing correctly (the notion of "natural" will be formalized in Section 3).

\section{Setting the Stage}

We begin with an informal description of the game at hand (as stated in the abstract). To facilitate our proofs, we will present a slightly more general version. Throughout, $\mathbb{Z}^{+}$will denote the set $\{1,2,3, \ldots\}$ of positive integers. For $n \in \mathbb{Z}^{+}$, we shall denote the set $\{1,2, \ldots, n\}$ simply by $[n]$.

Let $n \in \mathbb{Z}^{+}$and let $X \subseteq \mathbb{Z}^{+}$have cardinality $n$. The set $X$ is presented to Player A and Player B. Player A chooses an integer $\alpha \in X$ uniformly at random, but does not reveal $\alpha$ to Player B. However, Player B knows that Player A has chosen such an $\alpha$. Player B is permitted a total of $m \geq 1$ yes/no questions in sequence ( $m$ is revealed to Player B before the game commences), after which Player B must guess which number was chosen by Player A. Player B wins if she guesses $\alpha$. In the sequel, we shall denote this game by $\mathcal{G}(m, X)$; we agree to use the notation $\mathcal{G}(m, n)$ in case $X=[n]$. Our goal is to determine all possible $m$-question algorithms (from a canonical set of algorithms; more on this shortly) that will deliver the maximum probability of Player B winning $\mathcal{G}(m, X)$.

Our first task is to make further assumptions in order to force the game to terminate in a winner. To kick things off, we consider an example of a yes/no question that is, in some sense, very bad.

Example 1. Suppose that Player B asks, "Is the number you chose equal to the cardinality of the set of Fermat primes?" in the game $\mathcal{G}(m, n)$, where $n>4$. It is known only that there are at least 5 Fermat primes; it is not known if there are any more. So if Player A's chosen number is 5, then Player A simply (at present) does not know the answer to Player B's question, and the game ends in a stalemate.

In light of this example, we temporarily idealize Player A as follows (we shall later be able to dispense with this assumption):

Assumption 1. Player A has perfect knowledge, that is, Player A knows the truth value of $P$ for every proposition $P$.

Now, notice that any yes/no question asked by Player B can be phrased in the form, "Is it the case that $P$ ?" for some proposition $P$. This leads to an equivalent formulation of the game which is a bit more convenient for our purposes: 


\section{A. HAMMETT AND G. OMAN}

Assumption 2. All "questions" asked by Player B are merely propositions. Player A returns "true" if the proposition is true and "false" otherwise.

We now set up notation which will be heavily utilized throughout the remainder of this section.

Definition 1. Let $P$ be a proposition presented by Player $B$ in the game $\mathcal{G}(m, X)$. Then set

(1) $P^{0}:=\{i \in X:$ Player A returns "false" if he picked number $i\}$, and

(2) $P^{1}:=\{j \in X:$ Player A returns "true" if he picked number $j\}$.

To help the reader intuit this definition, we present two examples.

Example 2. Suppose Player $B$ presents $P:=$ "Denver is the capital of Colorado" in the game $\mathcal{G}(m, X)$. Then $P^{0}=\varnothing$ and $P^{1}=X$.

Example 3. Assume Player $B$ presents $P:=$ "The number you chose is prime" in the game $\mathcal{G}(m, 10)$. Then $P^{0}=\{1,4,6,8,9,10\}$ and $P^{1}=$ $\{2,3,5,7\}$.

We now transition to a probabilistic paradigm. Recall that the game $\mathcal{G}(m, X)$ begins with Player A choosing, uniformly at random, an integer $\alpha \in X$. The game concludes with Player B making a guess $\beta$ based upon the information she receives from Player A, and B wins the game if and only if $\alpha=\beta$. Throughout the remainder of this section, we shall denote Player A's choice by $\alpha$ and Player B's guess by $\beta$. In this setting, we regard $X$ as a probability space endowed with the uniform distribution

$$
\mathbb{P}(x)=\frac{1}{|X|} \text { for all } x \in X .
$$

Note that if Player B presents proposition $P$ to Player A, then (as both $P^{0}$ and $P^{1}$ are subsets of $\left.X\right) P^{0}$ and $P^{1}$ are events on the probability space $X$. Namely, $P^{0}$ is the event "Player A returns 'false' after receiving $P$," and $P^{1}$ the event "Player A returns 'true' after receiving $P$." Importantly, we do not assume that Player B knows precisely which elements $P^{0}$ and $P^{1}$ contain. We elaborate below.

Suppose Player B is playing $\mathcal{G}(1,10)$, and let $P$ be "The number you chose is prime" as in Example 3. Let $\mathcal{S}:=P^{i}$, where $i=0$ if Player A's response to $P$ was "false," and $i=1$ otherwise. Then all Player B knows for sure is that Player A's choice $\alpha \in[10]$ was uniformly random, and that in fact $\alpha \in \mathcal{S}=P^{i}$. What is the probability that Player B will now successfully guess $\alpha$ ? This really depends upon Player B's knowledge, given A's response $\mathcal{S}$. Suppose that $\mathcal{T}$ is a nonempty subset of [10] from which Player B will guess uniformly at random, given that $\alpha \in \mathcal{S}$. Let's refer to $\mathcal{T}$ 


\section{I'M THINKING OF A NUMBER ...}

as Player B's "guessing set." If Player B has absolutely no knowledge about which numbers between 1 and 10 are prime, then she will have no idea which elements belong to $\mathcal{S}$. So B may as well use guessing set $\mathcal{T}=$ [10], and the probability she will win equals $\frac{1}{10}<\frac{1}{|\mathcal{S}|}$. However, if B knows that $\{2,3,5\}$ are primes and $\{1,4\}$ are not (and nothing more about the integers in [10]), then things change. Indeed, if $\mathcal{S}=P^{0}$ (i.e. Player A answered "false") then Player B knows $\{1,4\} \subseteq \mathcal{S}$. So it is reasonable that B use guessing set $\mathcal{T}=\{1,4\}$, and the probability she will win equals $\frac{1}{6}=\frac{1}{|\mathcal{S}|}$. On the other hand, if $\mathcal{S}=P^{1}$, then B knows $\{2,3,5\} \subseteq \mathcal{S}$, so B should use guessing set $\mathcal{T}=\{2,3,5\}$. In this case, the probability she will win equals $\frac{1}{4}=\frac{1}{|\mathcal{S}|}$. Finally, if B knows precisely which numbers between 1 and 10 are prime, then she may use guessing set $\mathcal{T}=\mathcal{S}$ and B's probability of winning equals $\frac{1}{|\mathcal{S}|}$. Note that in each of these cases, Player B's probability of winning is at most $\frac{1}{|\mathcal{S}|}$. We generalize our observations above with a final assumption.

Assumption 3. After presenting all $m$ propositions to Player $A$ and receiving $A$ 's responses in the game $\mathcal{G}(m, X)$, Player $B$ selects a nonempty subset $\mathcal{T} \subseteq X$ (a "guessing set") from which she selects $\beta$ uniformly at random.

We are now ready to determine an upper bound on the probability of Player B winning $\mathcal{G}(m, X)$ (with Assumptions 1-3 above).

Theorem 1. The probability that Player $B$ wins the game $\mathcal{G}(m, X)$ is at most $\frac{\min \left(|X|, 2^{m}\right)}{|X|}$.

Proof. Player B will win $\mathcal{G}(m, X)$ with probability at most $1=\frac{|X|}{|X|}$. Thus

it suffices only to prove that the probability of winning is at most $\frac{2^{m}}{|X|}$. Let's assume that Player A has chosen $\alpha \in X$ uniformly at random, and fix an arbitrary set $\left\{P_{1}, \ldots, P_{m}\right\}$ of propositions to be presented by Player B. Let $\mathcal{W}$ denote the event, "Player B's guess $\beta$ is equal to $\alpha$." For $1 \leq i \leq m$, recall that $P_{i}^{0} \subseteq X$ denotes the event "Player A says 'false' after receiving $P_{i}$." Similarly, $P_{i}^{1}$ is the event "Player A says 'true' after receiving $P_{i}$." Then $\mathcal{W}$ occurs if and only if $P_{1}^{i_{1}} \cap P_{2}^{i_{2}} \cap \cdots \cap P_{m}^{i_{m}} \cap \mathcal{W}$ occurs for some $\left(i_{1}, i_{2}, \ldots, i_{m}\right) \in\{0,1\}^{m}$. Since for $\left(i_{1}, \ldots, i_{m}\right) \neq\left(j_{1}, \ldots, j_{m}\right)$, the events $P_{1}^{i_{1}} \cap \cdots \cap P_{m}^{i_{m}} \cap \mathcal{W}$ and $P_{1}^{j_{1}} \cap \cdots \cap P_{m}^{j_{m}} \cap \mathcal{W}$ are mutually exclusive, it follows that

$$
\mathbb{P}(\mathcal{W})=\sum_{\left(i_{1}, \ldots, i_{m}\right) \in\{0,1\}^{m}} \mathbb{P}\left(i_{1}, \ldots, i_{m}, \mathcal{W}\right)
$$




\section{A. HAMMETT AND G. OMAN}

where $\left(i_{1}, \ldots, i_{m}, \mathcal{W}\right)$ is the vector naming the event $P_{1}^{i_{1}} \cap \cdots \cap P_{m}^{i_{m}} \cap \mathcal{W}$. Since there are $2^{m}$ such vectors, it suffices to show that $\mathbb{P}(\mathbf{v}) \leq \frac{1}{|X|}$ for any such vector $\mathbf{v}$. Thus let $\mathbf{v}:=\left(i_{1}, \ldots, i_{m}, \mathcal{W}\right)$ be arbitrary. Observe that

$\mathbf{v}$ occurs if and only if $\alpha \in \mathcal{S}:=P_{1}^{i_{1}} \cap \ldots \cap P_{m}^{i_{m}}$ and Player B's guess $\beta$ equals $\alpha$.

Thus if $\mathcal{S}=\varnothing$, then $\mathbf{v}$ cannot occur, and $\mathbb{P}(\mathbf{v})=0<\frac{1}{|X|}$. Assume now that $\mathcal{S} \neq \varnothing$. Then

$$
\begin{aligned}
\mathbb{P}(\mathbf{v}) & =\mathbb{P}(\alpha \in \mathcal{S}) \cdot \mathbb{P}(\beta=\alpha \mid \alpha \in \mathcal{S}) \\
& =\frac{|\mathcal{S}|}{|X|} \cdot \frac{|\mathcal{S} \cap \mathcal{T}|}{|\mathcal{S}| \cdot|\mathcal{T}|}=\frac{|\mathcal{S} \cap \mathcal{T}|}{|\mathcal{T}|} \cdot \frac{1}{|X|} \leq \frac{1}{|X|}
\end{aligned}
$$

here, $\mathbb{P}(\beta=\alpha \mid \alpha \in \mathcal{S})$ denotes the conditional probability that $\beta=\alpha$ given that $\alpha \in \mathcal{S}$. To conclude the proof, we justify why $\mathbb{P}(\beta=\alpha \mid \alpha \in \mathcal{S})=$ $\frac{|\mathcal{S} \cap \mathcal{T}|}{|\mathcal{S}| \cdot|\mathcal{T}|}$. Since Player A's selection lies in $\mathcal{S}$, there are a total of $|\mathcal{S}| \cdot|\mathcal{T}|$ equally likely pairs $(\alpha, \beta)$ of possible choices by players $\mathrm{A}$ and $\mathrm{B}$, respectively. Player B will win provided the pair $(\alpha, \beta)$ satisfies $\alpha=\beta$, and there are $|\mathcal{S} \cap \mathcal{T}|$ such pairs. The proof is now complete.

The perceptive reader may have noticed the power of two appearing in the bound on $\mathbb{P}(\mathcal{W})$ above, and wondered if there is a relation between our work thus far and the binary search algorithm of computer science. Indeed there is. Without taking the reader too far afield, we mention simply that the binary search algorithm finds a specified key value in an array by repeatedly bisecting the array and making comparisons. For instance, suppose you want (your computer) to find the number 10 in the array $1,2,3,4,5,6,7,8,9,10$. Begin by taking the midpoint, 5, and comparing it with the desired value. Ten is bigger. Now repeat the process on the array $6,7,8,9,10$. The midpoint, 8 , is less than 10 . Repeat on the array 9,10 . Whether 9 or 10 is chosen as the next midpoint, the algorithm will, at worst, terminate with 10 on the fourth comparison. Indeed, it is not hard to show that any member of the original array can be found with at most 4 comparisons (this is the so-called worst case of the algorithm). Part of the purpose of the following section is to show that even with much less stringent assumptions (recall that Player B is free to ask Player A any question (proposition); the question need not even obviously relate to the game being played), Player B still cannot do any better than she can by adopting a natural set of additional rules for game play. We shall shortly introduce such a set of rules, and then find all strategies which maximize B's probability of winning in this modified probabilistic setting. 


\section{I'M THINKING OF A NUMBER ...}

We conclude this section with some simple applications of Theorem 1. Consider first the game $\mathcal{G}(1,2)$. Player B can assure a win by presenting the proposition, "The number you chose is 2" to Player A. If Player A returns "true," then Player B knows A's number is 2. If he returns "false," then Player B knows that A's number is 1 . Things change a bit if we consider $\mathcal{G}(1,3)$ instead. Theorem 1 tells us that the probability that Player B wins this game is at most $\frac{\min \left(3,2^{1}\right)}{3}=\frac{2}{3}$. Thus there does not exist a proposition $P$ that, regardless of the number chosen by Player A, will (after receiving Player A's response) allow Player B to deduce which number Player A chose. In fact, one need not resort to probabilistic methods in order to establish this; one needs only basic propositional logic. Suppose by way of contradiction that there exists such a proposition $P$. Let $\varphi$ be the statement, "Player A will choose a uniformly random number $\alpha \in$ [3] (observe that Player B knows $\varphi$ )." Then $\varphi \wedge P \models$ "Player A chose $i$ " and $\varphi \wedge \neg P \models$ "Player A chose $j$ " for some $i, j \in[3]$. Therefore $(\varphi \wedge P) \vee(\varphi \wedge$ $\neg P) \models$ "Player A chose either $i$ or $j$." But $(\varphi \wedge P) \vee(\varphi \wedge \neg P)$ is true. We deduce that Player A chose either $i$ or $j$. But of course, $|[3]|=3$, so this need not be so. We refer the interested reader to the bibliography for further reading on probability, logic, and algorithms.

\section{Main Results}

Now that we have Theorem 1 in our pocket, we are ready to give a canonical version of the game alluded to in the abstract. Throughout the remainder of this note, we shall assume $\mathcal{G}(m, X)$ to be as defined in Definition 2 below unless stated otherwise.

Definition 2 (The game $\mathcal{G}(m, X)$, canonical version). A finite, nonempty set $X \subseteq \mathbb{Z}^{+}$is presented to Players $A$ and $B$. Player $A$ randomly chooses a number $\alpha \in X$, but does not reveal $\alpha$ to Player B. Further, Player $B$ is given a positive integer $m$. For each $i \in[m]$, Player $B$ is to select some subset $B_{i} \subseteq X$ and presents it to Player $A$ (this is equivalent to Player $B$ asking Player $A$ if the number he chose is in $B_{i}$ ). After receiving $B_{i}$ from Player $B$, Player $A$ returns $A_{i}:=B_{i}$ if $\alpha \in B_{i}$ (this corresponds to an answer of "yes") and $A_{i}:=B_{i}^{c}$ (relative to $X$ ) if $\alpha \notin B_{i}$ (which corresponds to an answer of " $n o$ ") ${ }^{1}$. After Player $B$ has presented all $m$ sets to Player $A$ (and received all $m$ responses from Player $A$ ), Player $B$ attempts to guess the number Player A picked. Now set $A_{0}:=X$, and for $1 \leq i \leq m+1$, let $\mathscr{A}_{i}:=\bigcap_{0 \leq j<i} A_{j}$. We further impose the following on Player $B:^{2}$

(1) $B_{i} \subseteq \mathscr{A}_{i}$ for all $i \in[m]$, and

\footnotetext{
${ }^{1}$ Player $B$ presents $B_{i}$ to Player A after she has received $A_{i-1}$ from Player A.

${ }^{2}$ Player B loses if she does not follow (1) and (2) of Definition 2.
} 


\section{A. HAMMETT AND G. OMAN}

(2) Player B's guess is a member of $\mathscr{A}_{m+1}$ (that is, $\mathscr{A}_{m+1}$ is Player B's guessing set).

Note that at the $i$ th stage of the game (that is, the stage where Player B is about to select a set $B_{i}$ to present to Player A), Player B knows the elements of $\mathscr{A}_{i}$ and that $\alpha \in \mathscr{A}_{i}$. Thus she need not include any members of $\mathscr{A}_{i}^{c}$ in her set $B_{i}$ (ergo (1)). Similarly, after Player B has given all $m$ sets to Player A, she knows both the elements of $\mathscr{A}_{m+1}$ and that $\alpha \in \mathscr{A}_{m+1}$. Thus it makes no sense to guess outside of this set (hence (2)). Finally, observe that the game defined in Definition 2 above is a specific example of the game defined in the previous section, and therefore Theorem 1 applies in this context.

Recall from Theorem 1 that the probability of winning $\mathcal{G}(m, X)$ is at $\operatorname{most} \frac{\min \left(|X|, 2^{m}\right)}{|X|}$. We shall prove that this value can actually be achieved via the "canonical version" of the game just described. We leave the abstraction for a moment to present a concrete example.

Example 4. Consider the game $\mathcal{G}(2,6)$. To begin, Player A chooses some $\alpha \in[6] ;$ say $\alpha=4$. Now Player B is allotted two "questions" before guessing. Player $B$ presents the set $\{1,2,3\}$ to Player $A$, who then returns $\{4,5,6\}$. Player $B$ now presents $\{4,5\}$ to Player $A$, who returns $\{4,5\}$. Player $B$ guesses 4 , and wins the game.

We now turn our attention to determining Player B's strategy for achieving the maximum probability $\frac{\min \left(|X|, 2^{m}\right)}{|X|}$ of winning $\mathcal{G}(m, X)$. We begin with a definition and another example.

Definition 3. Let $X$ be a finite, nonempty subset of $\mathbb{Z}^{+}$, and let $m \geq 1$ be an integer. For each $i, 1 \leq i \leq m$, suppose $B_{i} \subseteq X$. Lastly, let $x_{0} \in X$. Then we call the sequence $\mathbf{g}:=\left(B_{1}, \ldots, B_{m}, x_{0}\right)$ a game vector of the game $\mathcal{G}(m, X)$. Further, we say that $\mathbf{g}$ is allowable in the game $\mathcal{G}(m, X)$ provided every $B_{i}$ satisfies (1) of Definition 2 and $x_{0}$ satisfies (2) of Definition 2. Lastly, $\mathbf{g}$ is winning if $\mathbf{g}$ is allowable and $x_{0}$ is the number chosen by Player A.

Example 5. Consider the game $\mathcal{G}(3,9)$ (that is, Player $A$ chooses some $\alpha \in[9]$ and Player $B$ is allotted 3 subsets of [9] before guessing), and suppose that Player $A$ chooses the number 1 . Set $B_{1}:=\{2,4,8\}, B_{2}:=$ $\{1,3,5,9\}, B_{2}^{\prime}:=\{1,2,5,9\}$, and $B_{3}:=\{1,5\}$. Then the game vector $\mathbf{g}:=\left(B_{1}, B_{2}, B_{3}, 5\right)$ is allowable and $\mathbf{g}^{\prime}:=\left(B_{1}, B_{2}, B_{3}, 1\right)$ is winning. However, the game vector $\mathbf{g}^{\prime \prime}:=\left(B_{1}, B_{2}, B_{3}, 6\right)$ is not allowable, since $6 \notin \mathscr{A}_{4}$. Finally, $\mathbf{g}^{\prime \prime \prime}:=\left(B_{1}, B_{2}^{\prime}, B_{3}, 1\right)$ is not allowable either, since $B_{2}^{\prime}$ is not a subset of $B_{1}$ or $B_{1}^{c}$ (hence $B_{2}^{\prime} \nsubseteq \mathscr{A}_{2}$ ).

We now establish a proposition which will be heavily utilized throughout the remainder of the paper. In what follows, the notation $\mathcal{G}_{\alpha}(m, X)$ will 


\section{I'M THINKING OF A NUMBER ...}

denote the game with $m$ questions on the finite, nonempty set $X \subseteq \mathbb{Z}^{+}$in which Player A chooses $\alpha$.

Proposition 1. Let $X \subseteq \mathbb{Z}^{+}$be finite and nonempty and let $m>1$ be an integer. Further, suppose that $B_{1}, \ldots, B_{m}$ are subsets of $X$ and that $x_{0} \in X$. Then the following hold:

(a) Let $A_{1}$ be Player $A$ 's response to $B_{1}$ in the game $\mathcal{G}_{\alpha}(m, X)$. Then $A_{1}$ is a finite subset of $\mathbb{Z}^{+}$containing $\alpha$. Thus the game $\mathcal{G}_{\alpha}(m-$ $\left.1, A_{1}\right)$ is well-defined.

(b) $\left(B_{1}, \ldots, B_{m}, x_{0}\right)$ is an allowable game vector of the game $\mathcal{G}_{\alpha}(m, X)$ if and only if $\left(B_{2}, \ldots, B_{m}, x_{0}\right)$ is an allowable game vector of the game $\mathcal{G}_{\alpha}\left(m-1, A_{1}\right)$.

(c) $\left(B_{1}, \ldots, B_{m}, x_{0}\right)$ is a winning game vector of the game $\mathcal{G}_{\alpha}(m, X)$ if and only if

$\left(B_{2}, \ldots, B_{m}, x_{0}\right)$ is a winning game vector of the game $\mathcal{G}_{\alpha}(m-$ $\left.1, A_{1}\right)$.

Proof. Assume that $X \subseteq \mathbb{Z}^{+}$is finite and nonempty and that $m>1$. Assume in addition that $B_{i} \subseteq X$ for $1 \leq i \leq m$ and that $x_{0} \in X$.

(a) By definition, Player A returns $A_{1}:=B_{1}$ if $\alpha \in B_{1}$ and $A_{1}:=X \backslash B_{1}$ otherwise. Since $X$ is finite, it follows in either case that $A_{1}$ is a finite subset of $\mathbb{Z}^{+}$. Moreover, $\alpha \in A_{1}$. Therefore, the game $\mathcal{G}_{\alpha}\left(m-1, A_{1}\right)$ is well-defined.

(b) Assume first that $\left(B_{1}, \ldots, B_{m}, x_{0}\right)$ is an allowable game vector of $\mathcal{G}_{\alpha}(m, X)$ and let $2 \leq i \leq m$ be arbitrary. Then by definition of "allowable," it follows that

$$
B_{i} \subseteq \mathscr{A}_{i}=\bigcap_{0 \leq j<i} A_{j} \subseteq A_{1} \quad \text { and } \quad x_{0} \in \mathscr{A}_{m+1}=\bigcap_{0 \leq j \leq m} A_{j} \subseteq A_{1} .
$$

Therefore, $B_{2} \cup B_{3} \cup \ldots \cup B_{m} \cup\left\{x_{0}\right\} \subseteq A_{1}$. We conclude that $\left(B_{2}, B_{3}, \ldots\right.$, $\left.B_{m}, x_{0}\right)$ is a game vector of the game $\mathcal{G}_{\alpha}\left(m-1, A_{1}\right)$. Now set $A_{1}^{\prime}:=A_{1}$ (this is the analog of $A_{0}$ in Definition 2), and for $2 \leq i \leq m$, let $A_{i}^{\prime}$ be Player A's response to $B_{i}$ in the game $\mathcal{G}_{\alpha}\left(m-1, A_{1}\right)$. Lastly, for $2 \leq i \leq m+1$, set $\mathscr{A}_{i}^{\prime}:=\bigcap_{1 \leq j<i} A_{j}$.

It follows immediately by definition of $\mathcal{G}_{\alpha}\left(m-1, A_{1}\right)$ that for $2 \leq i \leq m$,

$$
A_{i}^{\prime}= \begin{cases}B_{i}=A_{i} & \text { if } \alpha \in B_{i} \\ A_{1} \backslash B_{i} & \text { if } \alpha \notin B_{i} .\end{cases}
$$

We shall prove that

$$
\mathscr{A}_{i} \subseteq \mathscr{A}_{i}^{\prime} \text { for all } i, 2 \leq i \leq m+1
$$

MISSOURI J. OF MATH. SCI., SPRING 2016 


\section{A. HAMMETT AND G. OMAN}

Toward this end, choose $i$ with $2 \leq i \leq m+1$ and let $x \in \mathscr{A}_{i}$ be arbitrary. We prove that $x \in \mathscr{A}_{i}^{\prime}$. Pick $j$ with $1 \leq j<i$. We must show that $x \in A_{j}^{\prime}$. If $j=1$, the result is clear since $A_{1}^{\prime}=A_{1}$ and $\mathscr{A}_{i} \subseteq A_{1}$. Now assume that $2 \leq j<i$. As $x \in \mathscr{A}_{i}$, we have $x \in A_{j}$. Suppose first that $\alpha \in B_{j}$. Then (3.1) implies that $A_{j}=A_{j}^{\prime}$, hence $x \in A_{j}^{\prime}$. Assume now that $\alpha \notin B_{j}$. Then $A_{j}=X \backslash B_{j}$ and $A_{j}^{\prime}=A_{1} \backslash B_{j}$. Since $x \in A_{j}$, we conclude that $x \notin B_{j}$. Thus to prove that $x \in A_{j}^{\prime}$, it suffices to show that $x \in A_{1}$. Recall that $2 \leq i \leq m+1$ and that $x \in \mathscr{A}_{i}$. Thus $x \in A_{1}$ by definition of $\mathscr{A}_{i}$. This concludes the proof of (3.2). It is now easy to see that $\left(B_{2}, \ldots, B_{m}, x_{0}\right)$ is allowable: we simply need to check that $B_{i} \subseteq \mathscr{A}_{i}^{\prime}$ for $2 \leq i \leq m$ and that $x_{0} \in \mathscr{A}_{m+1}^{\prime}$. But this follows immediately from (3.2) and the assumption that $\left(B_{1}, \ldots, B_{m}, x_{0}\right)$ is allowable.

Now suppose that $\left(B_{2}, \ldots, B_{m}, x_{0}\right)$ is allowable in the game $\mathcal{G}_{\alpha}(m-$ $\left.1, A_{1}\right)$. By assumption,

$$
B_{1} \subseteq X=\mathscr{A}_{1}
$$

It follows from (3.1) that

$$
A_{i}^{\prime} \subseteq A_{i} \text { for } 1 \leq i \leq m \text { (that } A_{1}^{\prime} \subseteq A_{1} \text { is by definition). }
$$

Analogous to (3.2) above, we now prove that

$$
\mathscr{A}_{i}^{\prime} \subseteq \mathscr{A}_{i} \text { for all } i, 2 \leq i \leq m+1 .
$$

Let $2 \leq i \leq m+1$ be arbitrary. Then

$$
\mathscr{A}_{i}^{\prime}=\bigcap_{1 \leq j<i} A_{j}^{\prime} \subseteq \bigcap_{1 \leq j<i} A_{j}=X \cap\left(\bigcap_{1 \leq j<i} A_{j}\right)=\bigcap_{0 \leq j<i} A_{j}=\mathscr{A}_{i} ;
$$

the containment is immediate from (3.4) above. We conclude from (3.3), (3.5), and the assumption that $\left(B_{2}, \ldots, B_{m}, x_{0}\right)$ is allowable that $\left(B_{1}, \ldots\right.$, $\left.B_{m}, x_{0}\right)$ is an allowable game vector of $\mathcal{G}(m, X)$.

(c) This follows immediately from (b).

At long last, we are ready to establish the main result of this note via induction. In particular, we now have the machinery required to characterize all optimal strategies for Player B. Since the base case of the induction may be of independent interest, we single it out and prove it separately. First, we remind the reader that by Theorem 1, the probability that Player B wins game $\mathcal{G}(m, X)$ cannot exceed $\frac{\min \left(|X|, 2^{m}\right)}{|X|}$.

Proposition 2. Let $X$ be a finite, nonempty subset of $\mathbb{Z}^{+}$, and let $\alpha \in X$ be random. Now let $\mathbf{g}:=\left(B_{1}, x_{0}\right)$ be an arbitrary allowable game vector of 


\section{I'M THINKING OF A NUMBER ...}

the game $\mathcal{G}_{\alpha}(1, X)$. Finally, let $\mathcal{W}_{\mathbf{g}}$ be the event, " $\mathrm{g}$ is a winning vector of the game $\mathcal{G}_{\alpha}(1, X)$." Then

(a) $\mathbb{P}\left(\mathcal{W}_{\mathbf{g}}\right)=\frac{\min (|X|, 2)}{|X|}$ if and only if

(b) $\min (1,|X|-1) \leq\left|B_{1}\right| \leq \max (1,|X|-1)$.

Proof. Suppose first that $|X|=1$. Then (b) automatically holds, and the equivalence of (a) and (b) reduces to the assertion that $\mathrm{g}$ is a winning vector. Regardless of whether $B_{1}=\varnothing$ or $B_{1}=X$, Player A will return $X$. Since $\mathbf{g}$ is a game vector, it follows by definition that $x_{0} \in X$. Hence $x_{0}=\alpha$, and $\mathbf{g}$ is winning.

We now assume that $|X|>1$ and that Player B has presented $B_{1}$ to Player A. Let $\mathcal{B}_{1}$ be the event, "Player A returns $B_{1}$," and let $\mathcal{B}_{1}^{c}$ be the event, "Player A returns $B_{1}^{c}$." Since either $\mathcal{B}_{1}$ or $\mathcal{B}_{1}^{c}$ must occur and since these events are mutually exclusive, we deduce that

$$
\mathbb{P}\left(\mathcal{W}_{\mathbf{g}}\right)=\mathbb{P}\left(\mathcal{W}_{\mathbf{g}} \cap\left(\mathcal{B}_{1} \cup \mathcal{B}_{1}^{c}\right)\right)=\mathbb{P}\left(\mathcal{W}_{\mathbf{g}} \cap \mathcal{B}_{1}\right)+\mathbb{P}\left(\mathcal{W}_{\mathbf{g}} \cap \mathcal{B}_{1}^{c}\right) .
$$

Suppose now that (b) above fails. We shall prove that (a) fails too. It is easy to see that (b) fails if and only if $B_{1}=\varnothing$ or $B_{1}=X$. Suppose that $B_{1}=\varnothing$. Then $\mathcal{B}_{1}$ does not occur, and we deduce from (3.6) above that

$$
\mathbb{P}\left(\mathcal{W}_{\mathbf{g}}\right)=\mathbb{P}\left(\mathcal{W}_{\mathbf{g}} \cap \mathcal{B}_{1}^{c}\right)=\mathbb{P}\left(\mathcal{B}_{1}^{c}\right) \cdot \mathbb{P}\left(\mathcal{W}_{\mathbf{g}} \mid \mathcal{B}_{1}^{c}\right)=1 \cdot \frac{1}{|X|}<\frac{\min (|X|, 2)}{|X|},
$$

and we have shown that (a) fails. An analogous argument applies in case $B_{1}=X$.

Conversely, assume (b) holds. Then both $B_{1}$ and $B_{1}^{c}$ are nonempty. In this case, (3.6) becomes

$$
\begin{aligned}
\mathbb{P}\left(\mathcal{W}_{\mathbf{g}}\right) & =\mathbb{P}\left(\mathcal{W}_{\mathbf{g}} \cap \mathcal{B}_{1}\right)+\mathbb{P}\left(\mathcal{W}_{\mathbf{g}} \cap \mathcal{B}_{1}^{c}\right) \\
& =\mathbb{P}\left(\mathcal{B}_{1}\right) \cdot \mathbb{P}\left(\mathcal{W}_{\mathbf{g}} \mid \mathcal{B}_{1}\right)+\mathbb{P}\left(\mathcal{B}_{1}^{c}\right) \cdot \mathbb{P}\left(\mathcal{W}_{\mathbf{g}} \mid \mathcal{B}_{1}^{c}\right) \\
& =\frac{\left|B_{1}\right|}{|X|} \cdot \frac{1}{\left|B_{1}\right|}+\frac{\left|B_{1}^{c}\right|}{|X|} \cdot \frac{1}{\left|B_{1}^{c}\right|}=\frac{2}{|X|}=\frac{\min (|X|, 2)}{|X|}
\end{aligned}
$$

as required.

Now is a good time to reflect upon our results to this point in light of the examples given in the introduction. Let $n>1$ be arbitrary. Then in the game $\mathcal{G}(1, n)$ (as introduced in the introduction, with Player B asking a yes/no question to Player A), Player B can maximize her probability of winning by asking the following (seemingly naive) simple question: "Is the number you picked equal to 1?" We find this fact quite surprising. In fact, we can say a bit more: Player B maximizes her probability of winning 


\section{A. HAMMETT AND G. OMAN}

$\mathcal{G}(1, n)$ with question $Q$ if she knows a number $x \in[n]$ for which Player A answers "no" to $Q$ if he picked $x$ and a value $y \in[n]$ for which Player A responds with "yes" to $Q$ if he picked $y$. We now present the main result of this paper.

Theorem 2. Let $X$ be a finite, nonempty subset of $\mathbb{Z}^{+}$, and let $\alpha \in X$ be random. Now let $\mathbf{g}:=\left(B_{1}, \ldots, B_{m}, x_{0}\right)$ be an arbitrary allowable game vector of the game $\mathcal{G}_{\alpha}(m, X)$. We remind the reader that $A_{0}:=X$ and for each $i \in[m], A_{i}$ denotes Player $A$ 's response to the set $B_{i}$ presented by Player B. Finally, $\mathscr{A}_{i}:=\bigcap_{0 \leq j<i} A_{j}$ for $i \in[m+1]$. Now let $\mathcal{W}_{\mathbf{g}}\left(\mathcal{G}_{\alpha}(m, X)\right)$ be the event, "g is a winning vector of the game $\mathcal{G}_{\alpha}(m, X)$." Then

(a) $\mathbb{P}\left(\mathcal{W}_{\mathbf{g}}\left(\mathcal{G}_{\alpha}(m, X)\right)\right)=\frac{\min \left(|X|, 2^{m}\right)}{|X|}$ if and only if

(b) $\min \left(2^{m-i},\left|\mathscr{A}_{i}\right|-2^{m-i}\right) \leq\left|B_{i}\right| \leq \max \left(2^{m-i},\left|\mathscr{A}_{i}\right|-2^{m-i}\right)$ for all $i$, $1 \leq i \leq m$.

Proof. We proceed by induction on $m$. Thus suppose the theorem is true for all $k<m$. If $m=1$, then we are done by Proposition 2. Therefore, we may suppose that

$$
m>1 .
$$

Now set

$$
\mathbf{g}^{\prime}:=\left(B_{2}, \ldots, B_{m}, x_{0}\right) .
$$

As in the proof of Proposition 2, we let $\mathcal{B}_{1}$ denote the event, "Player A returns $B_{1}$ after being presented with $B_{1}$," and $\mathcal{B}_{1}^{c}$ name the event, "Player A returns $B_{1}^{c}$ after being presented with $B_{1}$." We now consider two cases.

Case 1: $B_{1}=\varnothing$ or $B_{1}=X$. Then $\mathbb{P}\left(\mathcal{B}_{1}^{c}\right)=1$ or $\mathbb{P}\left(\mathcal{B}_{1}\right)=1$, respectively, and we have

$$
\begin{aligned}
& \mathbb{P}\left(\mathcal{W}_{\mathbf{g}}\left(\mathcal{G}_{\alpha}(m, X)\right)\right) \\
& =\left\{\begin{array}{l}
\mathbb{P}\left(\mathcal{W}_{\mathbf{g}}\left(\mathcal{G}_{\alpha}(m, X)\right) \cap \mathcal{B}_{1}^{c}\right)=\mathbb{P}\left(\mathcal{W}_{\mathbf{g}}\left(\mathcal{G}_{\alpha}(m, X)\right) \mid \mathcal{B}_{1}^{c}\right) \text { if } B_{1}=\varnothing, \\
\mathbb{P}\left(\mathcal{W}_{\mathbf{g}}\left(\mathcal{G}_{\alpha}(m, X)\right) \cap \mathcal{B}_{1}\right)=\mathbb{P}\left(\mathcal{W}_{\mathbf{g}}\left(\mathcal{G}_{\alpha}(m, X)\right) \mid \mathcal{B}_{1}\right) \text { if } B_{1}=X .
\end{array}\right.
\end{aligned}
$$

If $B_{1}=\varnothing$, then Proposition 1 implies that $\mathbb{P}\left(\mathcal{W}_{\mathbf{g}}\left(\mathcal{G}_{\alpha}(m, X)\right) \mid \mathcal{B}_{1}^{c}\right)=$ $\mathbb{P}\left(\mathcal{W}_{\mathbf{g}^{\prime}}\left(\mathcal{G}_{\alpha}(m-1, X)\right)\right)$. Similarly, if $B_{1}=X$, then $\mathbb{P}\left(\mathcal{W}_{\mathbf{g}}\left(\mathcal{G}_{\alpha}(m, X)\right) \mid \mathcal{B}_{1}\right)=$ $\mathbb{P}\left(\mathcal{W}_{\mathbf{g}^{\prime}}\left(\mathcal{G}_{\alpha}(m-1, X)\right)\right)$. In either case, $(3.11)$ reduces to

$$
\mathbb{P}\left(\mathcal{W}_{\mathbf{g}}\left(\mathcal{G}_{\alpha}(m, X)\right)\right)=\mathbb{P}\left(\mathcal{W}_{\mathbf{g}^{\prime}}\left(\mathcal{G}_{\alpha}(m-1, X)\right)\right) .
$$

We are ready to establish the equivalence of (a) and (b). Suppose first that 


$$
\mathbb{P}\left(\mathcal{W}_{\mathbf{g}}\left(\mathcal{G}_{\alpha}(m, X)\right)\right)=\frac{\min \left(|X|, 2^{m}\right)}{|X|} .
$$

For all $i, 1 \leq i \leq m$, we must prove that

$$
\min \left(2^{m-i},\left|\mathscr{A}_{i}\right|-2^{m-i}\right) \leq\left|B_{i}\right| \leq \max \left(2^{m-i},\left|\mathscr{A}_{i}\right|-2^{m-i}\right) .
$$

We deduce from (3.12) and (3.13) that

$$
\mathbb{P}\left(\mathcal{W}_{\mathbf{g}^{\prime}}\left(\mathcal{G}_{\alpha}(m-1, X)\right)\right)=\frac{\min \left(|X|, 2^{m}\right)}{|X|} .
$$

Recall from Theorem 1 that $\mathbb{P}\left(\mathcal{W}_{\mathbf{g}^{\prime}}\left(\mathcal{G}_{\alpha}(m-1, X)\right)\right) \leq \frac{\min \left(|X|, 2^{m-1}\right)}{|X|}$. This fact along with (3.15) implies

$$
\mathbb{P}\left(\mathcal{W}_{\mathbf{g}^{\prime}}\left(\mathcal{G}_{\alpha}(m-1, X)\right)\right)=\frac{\min \left(|X|, 2^{m}\right)}{|X|}=\frac{\min \left(|X|, 2^{m-1}\right)}{|X|} .
$$

It is patent from (3.16) that

$$
|X| \leq 2^{m-1} .
$$

We now prove that (3.14) holds for all $i, 1 \leq i \leq m$. When $i=1,(3.14)$ follows immediately from (3.17) above. Set $A_{1}^{\prime}:=X$ and for each $i$ with $2 \leq i \leq m$, let $A_{i}^{\prime}$ be Player A's response to $B_{i}$ in the game $\mathcal{G}_{\alpha}(m-1, X)$. Then we deduce from (3.16) and the inductive hypothesis that

$$
\min \left(2^{m-i},\left|\mathscr{A}_{i}^{\prime}\right|-2^{m-i}\right) \leq\left|B_{i}\right| \leq \max \left(2^{m-i},\left|\mathscr{A}_{i}^{\prime}\right|-2^{m-i}\right) \text { for } 2 \leq i \leq m,
$$

where $\mathscr{A}_{i}^{\prime}$ is defined as in the proof of Proposition 1. Since $B_{1}=\varnothing$ or $B_{1}=X$, it follows that $A_{1}=X$. Hence

$A_{i}^{\prime}=A_{i}$ for each $i, 1 \leq i \leq m$. Thus also $\mathscr{A}_{i}^{\prime}=\mathscr{A}_{i}$ for each $i, 2 \leq i \leq m$.

Combining (3.18) and (3.19), we see that $\min \left(2^{m-i},\left|\mathscr{A}_{i}\right|-2^{m-i}\right) \leq\left|B_{i}\right| \leq$ $\max \left(2^{m-i},\left|\mathscr{A}_{i}\right|-2^{m-i}\right)$ for $2 \leq i \leq m$, and (3.14) has been established for all $i$.

Conversely, assume that (3.14) holds for $1 \leq i \leq m$. We shall prove that $\mathbb{P}\left(\mathcal{W}_{\mathbf{g}}\left(\mathcal{G}_{\alpha}(m, X)\right)\right)=\frac{\min \left(|X|, 2^{m}\right)}{|X|}$. It follows immediately from (3.14), (3.19), and the inductive hypothesis that

$$
\mathbb{P}\left(\mathcal{W}_{\mathbf{g}^{\prime}}\left(\mathcal{G}_{\alpha}(m-1, X)\right)\right)=\frac{\min \left(|X|, 2^{m-1}\right)}{|X|} .
$$




\section{A. HAMMETT AND G. OMAN}

We deduce from (3.12) that

$$
\mathbb{P}\left(\mathcal{W}_{\mathbf{g}}\left(\mathcal{G}_{\alpha}(m, X)\right)\right)=\frac{\min \left(|X|, 2^{m-1}\right)}{|X|} .
$$

To conclude the Case 1 proof, it suffices to show that $|X| \leq 2^{m-1}$. Again, we remind the reader that our Case 1 assumption is that $B_{1}=\varnothing$ or $B_{1}=X$. If $B_{1}=\varnothing$, then $|X| \leq 2^{m-1}$ follows immediately from the first inequality in (3.14) above (with $i=1$ ). In case $B_{1}=X$, we deduce $|X| \leq 2^{m-1}$ from the right-hand $i=1$ inequality in (3.14). This concludes the proof in Case 1.

Case 2: $\varnothing \subsetneq B_{1} \subsetneq X$. In this case, Theorem 1, Proposition 1, and the argument used in the proof of Proposition 2 (see (3.6) and (3.8)) imply that

$$
\begin{aligned}
& \mathbb{P}\left(\mathcal{W}_{\mathbf{g}}\left(\mathcal{G}_{\alpha}(m, X)\right)\right) \\
& =\mathbb{P}\left(\mathcal{B}_{1}\right) \cdot \mathbb{P}\left(\mathcal{W}_{\mathbf{g}}\left(\mathcal{G}_{\alpha}(m, X)\right) \mid \mathcal{B}_{1}\right)+\mathbb{P}\left(\mathcal{B}_{1}^{c}\right) \cdot \mathbb{P}\left(\mathcal{W}_{\mathbf{g}}\left(\mathcal{G}_{\alpha}(m, X)\right) \mid \mathcal{B}_{1}^{c}\right) \\
& =\mathbb{P}\left(\mathcal{B}_{1}\right) \cdot \mathbb{P}\left(\mathcal{W}_{\mathbf{g}^{\prime}}\left(\mathcal{G}_{\alpha}\left(m-1, B_{1}\right)\right)\right)+\mathbb{P}\left(\mathcal{B}_{1}^{c}\right) \cdot \mathbb{P}\left(\mathcal{W}_{\mathbf{g}^{\prime}}\left(\mathcal{G}_{\alpha}\left(m-1, B_{1}^{c}\right)\right)\right) \\
& \leq \frac{\left|B_{1}\right|}{|X|} \cdot \frac{\min \left(\left|B_{1}\right|, 2^{m-1}\right)}{\left|B_{1}\right|}+\frac{\left|B_{1}^{c}\right|}{|X|} \cdot \frac{\min \left(\left|B_{1}^{c}\right|, 2^{m-1}\right)}{\left|B_{1}^{c}\right|} \\
& =\frac{\min \left(\left|B_{1}\right|, 2^{m-1}\right)}{|X|}+\frac{\min \left(\left|B_{1}^{c}\right|, 2^{m-1}\right)}{|X|} \\
& \leq \frac{\min \left(|X|, 2^{m}\right)}{|X|}
\end{aligned}
$$

Assume first that $\mathbb{P}\left(\mathcal{W}_{\mathbf{g}}\left(\mathcal{G}_{\alpha}(m, X)\right)\right)=\frac{\min \left(|X|, 2^{m}\right)}{|X|}$. Then equality holds throughout (3.22). It follows (regardless of whether $A_{1}=B_{1}$ or $A_{1}=B_{1}^{c}$ ) that

$$
\mathbb{P}\left(\mathcal{W}_{\mathbf{g}^{\prime}}\left(\mathcal{G}_{\alpha}\left(m-1, A_{1}\right)\right)\right)=\frac{\min \left(\left|A_{1}\right|, 2^{m-1}\right)}{\left|A_{1}\right|} .
$$

For $2 \leq i \leq m$, the inductive hypothesis yields

$$
\min \left(2^{m-i},\left|\mathscr{A}_{i}^{\prime}\right|-2^{m-i}\right) \leq\left|B_{i}\right| \leq \max \left(2^{m-i},\left|\mathscr{A}_{i}^{\prime}\right|-2^{m-i}\right),
$$

Invoking (3.2) and (3.5) of the proof of Proposition 1, (3.24) becomes

$$
\min \left(2^{m-i},\left|\mathscr{A}_{i}\right|-2^{m-i}\right) \leq\left|B_{i}\right| \leq \max \left(2^{m-i},\left|\mathscr{A}_{i}\right|-2^{m-i}\right)
$$

for $2 \leq i \leq m$. To complete the implication (a) $\Longrightarrow(\mathrm{b})$, we need only show that (3.25) also holds when $i=1$. Suppose not. Then either (1) $\left|B_{1}\right|<$ $2^{m-1}$ and $\left|B_{1}\right|<|X|-2^{m-1}$ or $(2)\left|B_{1}\right|>2^{m-1}$ and $\left|B_{1}\right|>|X|-2^{m-1}$. 


\section{I'M THINKING OF A NUMBER ...}

In either case, $(3.22)$ implies that $\mathbb{P}\left(\mathcal{W}_{\mathbf{g}}\left(\mathcal{G}_{\alpha}(m, X)\right)\right)<\frac{\min \left(|X|, 2^{m}\right)}{|X|}$, and we have a contradiction to our assumption.

Conversely, suppose that (3.25) holds for all $i, 1 \leq i \leq m$. Then by (3.2) and (3.5) of the proof of Proposition 1, we obtain (3.24) for $2 \leq i \leq m$. For the reader's convenience, we restate (3.22):

$$
\begin{aligned}
& \mathbb{P}\left(\mathcal{W}_{\mathbf{g}}\left(\mathcal{G}_{\alpha}(m, X)\right)\right) \\
& =\mathbb{P}\left(\mathcal{B}_{1}\right) \cdot \mathbb{P}\left(\mathcal{W}_{\mathbf{g}}\left(\mathcal{G}_{\alpha}(m, X)\right) \mid \mathcal{B}_{1}\right)+\mathbb{P}\left(\mathcal{B}_{1}^{c}\right) \cdot \mathbb{P}\left(\mathcal{W}_{\mathbf{g}}\left(\mathcal{G}_{\alpha}(m, X)\right) \mid \mathcal{B}_{1}^{c}\right) \\
& =\mathbb{P}\left(\mathcal{B}_{1}\right) \cdot \mathbb{P}\left(\mathcal{W}_{\mathbf{g}^{\prime}}\left(\mathcal{G}_{\alpha}\left(m-1, B_{1}\right)\right)\right)+\mathbb{P}\left(\mathcal{B}_{1}^{c}\right) \cdot \mathbb{P}\left(\mathcal{W}_{\mathbf{g}^{\prime}}\left(\mathcal{G}_{\alpha}\left(m-1, B_{1}^{c}\right)\right)\right) \\
& \leq \frac{\left|B_{1}\right|}{|X|} \cdot \frac{\min \left(\left|B_{1}\right|, 2^{m-1}\right)}{\left|B_{1}\right|}+\frac{\left|B_{1}^{c}\right|}{|X|} \cdot \frac{\min \left(\left|B_{1}^{c}\right|, 2^{m-1}\right)}{\left|B_{1}^{c}\right|} \\
& =\frac{\min \left(\left|B_{1}\right|, 2^{m-1}\right)}{|X|}+\frac{\min \left(\left|B_{1}^{c}\right|, 2^{m-1}\right)}{|X|} \\
& \leq \frac{\min \left(|X|, 2^{m}\right)}{|X|}
\end{aligned}
$$

As (3.24) holds for $2 \leq i \leq m$, the inductive hypothesis allows us to replace the first inequality sign above with equality. We conclude from (3.25) above (with $i=1$ ) that either (1) $\left|B_{1}\right| \leq 2^{m-1}$ and $\left|B_{1}^{c}\right| \leq 2^{m-1}$ or (2) $2^{m-1} \leq$ $\left|B_{1}\right|$ and $2^{m-1} \leq\left|B_{1}^{c}\right|$. In either case, we can replace the second inequality with equality, and the proof is complete.

We have determined the sets $B_{i} \subseteq X$ which maximize Player B's probability of winning $\mathcal{G}_{\alpha}(m, X)$. It remains to verify that Player B can, in fact, effectively find an allowable game vector $\left(B_{1}, \ldots, B_{m}, x_{0}\right)$ which satisfies (b) of Theorem 2.

Proposition 3. Consider the game $\mathcal{G}_{\alpha}(m, X)$, where $X \subseteq \mathbb{Z}^{+}$is finite and nonempty and $m \geq 1$. Then Player $B$ can effectively choose ${ }^{3}$ subsets $B_{1}, \ldots, B_{m}$ of $X$ and $x_{0} \in X$ such that

(a) $\left(B_{1}, \ldots, B_{m}, x_{0}\right)$ is an allowable game vector, and

(b) $\min \left(2^{m-i},\left|\mathscr{A}_{i}\right|-2^{m-i}\right) \leq\left|B_{i}\right| \leq \max \left(2^{m-i},\left|\mathscr{A}_{i}\right|-2^{m-i}\right)$ for all $i$, $1 \leq i \leq m$.

Proof. Fix $k$ with $1 \leq k \leq m$, and suppose that for each $i<k$, Player B has chosen $B_{i} \subseteq \mathscr{A}_{i}$ satisfying (b) and Player A has returned $A_{i}$ to her. Then of course, Player B knows precisely which elements of $X$ belong to $\mathscr{A}_{k}$. We claim that Player B can effectively choose $B_{k} \subseteq \mathscr{A}_{k}$ satisfying (b). We consider two cases.

\footnotetext{
${ }^{3}$ That is, there exists an algorithm by which Player $\mathrm{B}$ can choose $B_{1}, \ldots, B_{m}, x_{0}$ satisfying (a) and (b) regardless of which $\alpha$ was chosen by Player A.
}

MISSOURI J. OF MATH. SCI., SPRING 2016 


\section{A. HAMMETT AND G. OMAN}

Case 1: $\left|\mathscr{A}_{k}\right|-2^{m-k}<0$. Then Player B can take $B_{k}:=\varnothing$.

Case 2: $\left|\mathscr{A}_{k}\right|-2^{m-k} \geq 0$. Then Player B can choose any subset of $\mathscr{A}_{k}$ of size $\left|\mathscr{A}_{k}\right|-2^{m-k}$.

After choosing the sets $B_{1}, \ldots, B_{m}$ as above, it remains to show that Player B can effectively select $x_{0} \in \mathscr{A}_{m+1}$. Since $\alpha \in \mathscr{A}_{m+1}$, we have $\mathscr{A}_{m+1} \neq \varnothing$. Thus Player B can simply select her favorite element of $\mathscr{A}_{m+1}$ (as above, Player B knows exactly which elements of $X$ are members of $\mathscr{A}_{m+1}$ ), and the proof is complete.

\section{Some Consequences}

We conclude the paper with several consequences of the results of the previous section.

Corollary 1. Player $B$ has a winning strategy in the game $\mathcal{G}_{\alpha}(m, X)$ if and only if $m \geq \log _{2}|X|$.

Proof. Player B has a winning strategy in the game $\mathcal{G}_{\alpha}(m, X)$ if and only if $\frac{\min \left(|X|, 2^{m}\right)}{|X|}=1$ if and only if $2^{m} \geq|X|$ if and only if $m \geq \log _{2}|X|$.

It is natural to ask if it is possible to eliminate "min" and "max" from the formulation of (b) of Theorem 2. Indeed it is. We shall require the following simple lemma.

Lemma 1. Let $\left(B_{1}, \ldots, B_{m}, x_{0}\right)$ be an allowable game vector of the game $\mathcal{G}_{\alpha}(m, X)$ such that $\min \left(2^{m-1},|X|-2^{m-1}\right) \leq\left|B_{1}\right| \leq \max \left(2^{m-1},|X|-\right.$ $\left.2^{m-1}\right)$. Then $2^{m} \geq|X|$ if and only if $2^{m-1} \geq\left|A_{1}\right|$.

Proof. Suppose first that $2^{m} \geq|X|$. Then $2^{m-1} \geq|X|-2^{m-1}$, and hence $|X|-2^{m-1} \leq\left|B_{1}\right| \leq 2^{m-1}$. If $A_{1}=B_{1}$, then $2^{m-1} \geq\left|B_{1}\right|=\left|A_{1}\right|$, as required. Otherwise, $A_{1}=B_{1}^{c}$. Since $|X|-2^{m-1} \leq\left|B_{1}\right|$, we deduce that $\left|A_{1}\right|=|X|-\left|B_{1}\right| \leq 2^{m-1}$.

Now assume that $2^{m} \leq|X|$. Then $2^{m-1} \leq|X|-2^{m-1}$, and thus $2^{m-1} \leq$ $\left|B_{1}\right| \leq|X|-2^{m-1}$. As above, if $A_{1}=B_{1}$, then $2^{m-1} \leq\left|A_{1}\right|$. Suppose $A_{1}=B_{1}^{c}$. Since $\left|B_{1}\right| \leq|X|-2^{m-1}$, we obtain $\left|A_{1}\right|=|X|-\left|B_{1}\right| \geq 2^{m-1}$. This concludes the proof.

Corollary 2. Let $X \subseteq \mathbb{Z}^{+}$be finite and nonempty, $m \in \mathbb{Z}^{+}$, and $\mathrm{g}:=$ $\left(B_{1}, \ldots, B_{m}, x_{0}\right)$ be an allowable game vector of the game $\mathcal{G}_{\alpha}(m, X)$. Then the following hold:

(a) If $2^{m} \geq|X|$, then $\mathbb{P}\left(\mathcal{W}_{\mathbf{g}}\left(\mathcal{G}_{\alpha}(m, X)\right)\right)=1$ if and only if $\left|\mathscr{A}_{i}\right|-2^{m-i} \leq$ $\left|B_{i}\right| \leq 2^{m-i}$ for $1 \leq i \leq m$, and

(b) If $2^{m} \leq|X|$, then $\mathbb{P}\left(\mathcal{W}_{\mathbf{g}}\left(\mathcal{G}_{\alpha}(m, X)\right)\right)=\frac{2^{m}}{|X|}$ if and only if $2^{m-i} \leq$ $\left|B_{i}\right| \leq\left|\mathscr{A}_{i}\right|-2^{m-i}$ for $1 \leq i \leq m$. 


\section{I'M THINKING OF A NUMBER ...}

Proof. Let $X, m$, and $\mathbf{g}$ be as stated. We proceed by induction on $m$. Thus, we assume that the corollary holds for all $k<m$, and prove that it holds for $m$. If $m=1$, then (a) and (b) follow immediately from Theorem 2. Now suppose that $m>1$. By Theorem 2, it suffices to establish only the forward implications.

We prove only (a), as the proof of (b) is similar. Suppose $2^{m} \geq|X|$ and $\mathbb{P}\left(\mathcal{W}_{\mathbf{g}}\left(\mathcal{G}_{\alpha}(m, X)\right)\right)=1$. Then $|X|-2^{m-1} \leq 2^{m-1}$. By Theorem 2 , it follows that $|X|-2^{m-1} \leq\left|B_{1}\right| \leq 2^{m-1}$. Lemma 1 tells us that $2^{m-1} \geq\left|A_{1}\right|$. Recall from (3.2) and (3.5) of the proof of Proposition 1 that $\mathscr{A}_{i}=\mathscr{A}_{i}^{\prime}$ for $2 \leq i \leq m+1$. This fact along with the inductive hypothesis yields that $\left|\mathscr{A}_{i}\right|-2^{m-i} \leq\left|B_{i}\right| \leq 2^{m-i}$ for $2 \leq i \leq m$. The proof is now complete.

It is immediate from Corollary 2 that in the game $\mathcal{G}_{\alpha}(1, X),|X|>1$, Player B maximizes her probability of winning if and only if she chooses $B_{1} \subseteq X$ and $x_{0} \in X$ such that $\left(B_{1}, x_{0}\right)$ is allowable and $1 \leq\left|B_{1}\right| \leq$ $|X|-1$. Thus (for large values of $|X|$ ) there are subsets $B_{1} \subseteq X$ of many different cardinalities which maximize B's probability of winning $\mathcal{G}_{\alpha}(1, X)$. Moreover, in general, Player B has some freedom in choosing the sizes of the sets $B_{i}$ to present to Player A, even in the case when $m=\left\lceil\log _{2}|X|\right\rceil$. For instance, consider the game $\mathcal{G}_{\alpha}(5,19)$. Then (again, employing a winning strategy) Player B can begin by choosing any $B_{1} \subseteq[19]$ with the property that $3 \leq\left|B_{1}\right| \leq 16$.

We end this note by determining conditions under which Player B has no freedom in choosing the cardinalities of the sets $B_{1}, \ldots, B_{m}$, where $\left(B_{1}, \ldots, B_{m}, x_{0}\right)$ is a game vector which maximizes B's probability of win$\operatorname{ning} \mathcal{G}_{\alpha}(m, X)$.

Corollary 3. Let $X$ be a finite, nonempty subset of $\mathbb{Z}^{+}$and let $\mathrm{g}:=$ $\left(B_{1}, \ldots, B_{m}, x_{0}\right)$ be a game vector of the game $\mathcal{G}_{\alpha}(m, X)$ which maximizes Player B's probability of winning (that is, $\left.\mathbb{P}\left(\mathcal{W}_{\mathbf{g}}\left(\mathcal{G}_{\alpha}(m, X)\right)\right)=\frac{\min \left(|X|, 2^{m}\right)}{|X|}\right)$. Then the cardinalities of the sets $B_{i}$ are uniquely determined if and only if $|X|=2^{m}$ (in which case Player B has a winning strategy by Corollary 1).

Proof. Let $\mathbf{g}:=\left(B_{1}, \ldots, B_{m}, x_{0}\right)$ be an arbitrary game vector which maximizes the probability of Player B winning $\mathcal{G}_{\alpha}(m, X)$ (in particular, $\mathbf{g}$ is allowable). Assume first that the cardinalities of the sets $B_{i}$ are uniquely determined. Then Theorem 2 implies that $\min \left(2^{m-1},|X|-2^{m-1}\right) \leq\left|B_{1}\right| \leq$ $\max \left(2^{m-1},|X|-2^{m-1}\right)$. We claim that $2^{m-1}=|X|-2^{m-1}$. Otherwise, it is easy to see that there exist integers $n_{1} \neq n_{2}$ satisfying both

$$
\begin{gathered}
0 \leq n_{i} \leq|X|, \text { and } \\
\min \left(2^{m-1},|X|-2^{m-1}\right) \leq n_{i} \leq \max \left(2^{m-1},|X|-2^{m-1}\right)
\end{gathered}
$$

MISSOURI J. OF MATH. SCI., SPRING 2016 


\section{A. HAMMETT AND G. OMAN}

for $i=1,2$. But then both $\left|B_{1}\right|=n_{1}$ and $\left|B_{1}\right|=n_{2}$ are possible. This gives us a contradiction to the uniqueness of $\left|B_{1}\right|$. Thus $2^{m-1}=|X|-2^{m-1}$, and $|X|=2^{m}$.

Conversely, suppose that $|X|=2^{m}$. Then it follows immediately from Corollary 2 that $\left|B_{i}\right|=2^{m-i}$ for $1 \leq i \leq m$.

\section{References}

[1] P. Billingsley, Probability and Measure, Wiley, Hoboken, 2012.

[2] H. Enderton, A Mathematical Introduction to Logic, Academic Press, New York - London, 1972.

[3] K. Rosen, Discrete Mathematics and Its Applications, seventh edition, McGraw-Hill, New York, 2012.

MSC2010: 03B05, 60B99

Key words and phrases: event, conditional probability, probability

(Adam Hammett) Department of Science and Mathematics, Cedarville University, Cedarville, OH 45314, USA

E-mail address: ahammett@cedarville.edu

(Greg Oman) Department of Mathematics, The University of Colorado, Colorado Springs, CO 80918, USA

E-mail address: goman@uccs.edu 